\title{
Der Stellenwert der postoperativen Übungsbehandlung für das Outcome der distalen Radiusfraktur
}

\author{
Axel Lohsträter, Silvia Germann
}

\section{Zusammenfassung}

Hintergrund: Die vorgestellte Arbeit untersuchte im Rahmen einer prospektiv randomisierten Studie die Frage nach dem Stellenwert der konsequenten und strukturierten Übungsbehandlung auf das Outcome der distalen Radiusfraktur. Patienten und Methoden: Von 380 konsekutiven Patienten nach distaler Radiusfraktur konnten aufgrund der Ein-/Ausschlusskriterien 198 in die Studie eingeschlossen und einer Interventionsgruppe (gezieltes Management) oder einer Kontrollgruppe (D-Arzt-Verfahren) randomisiert zugeteilt werden. In der Interventionsgruppe erhielten die Behandler Empfehlungen zu Art, Umfang und Ausmaß der durchzuführenden Übungsbehandlung. Die Nachbehandlungsempfehlung wurde durch die VBG in der Umsetzung im Sinne eines Monitorings begleitet. Die Patienten wurden per DV-gestützter, externer Zufallsauswahl randomisiert. Die Signifikanztests wurden mit einem Signifikanzniveau von 5\% durchgeführt. Ergebnisse: Die Ergebnisse der Studie zeigten in der Interventionsgruppe ein geringeres Maß an verordneter Therapie. Diese wurde jedoch strukturierter und in kürzerer Zeit erbracht. Im Outcome, gemessen in der Minderung der Erwerbsfähigkeit (MdE), zeigte sich, bei kürzerer Arbeitsunfähigkeitszeit, in der Interventionsgruppe nur in 12,3\% der Fälle eine MdE. In der Kontrollgruppe wurde, trotz scheinbar längerer Nachbehandlungsphase, in 27,4\% der Fälle eine MdE festgestellt. Schlussfolgerung: Das Out- come (MdE) ist in der Interventionsgruppe signifikant besser. Höhere Therapiekosten sind in der Interventionsgruppe nicht zu beobachten. Untersuchungen, die Intensität, Dauer und Wert von Physio- und Ergotherapie beleuchten, sind bisher nicht publiziert. Die strukturierte Nachbehandlung im Sinne einer kombinierten Therapie wird in ihrer Bedeutung daher noch unterschätzt. Insbesondere bei der Verordnung von Ergotherapie fallen deutliche Defizite auf. Eine unterstützende Steuerung scheint daher geboten. Die Kombination beider Therapieverfahren sichert im Zusammenwirken aller Beteiligten einen größeren Behandlungserfolg.

\section{The Relevance of Postoperative Tutorial Treatment on the Outcome of Distal Radius Fractures}

Background: The relevance of consequent and structured tutorial treatment on the outcome of distal radius fractures was examined in the presented project within a prospective randomised study. Patients and Methods: On the basis of assessment criteria, 198 patients out of 380 consecutive distal fracture patients could be included into the study and assigned randomly to either a intervention group (focused management) or a control group (transition physician process). Recommendations about characteristics, extent and degree of the conducting practice treatment were given to the care-givers within the intervention group. Post-treatment recommenda- tions were evaluated by the cost unit. The above-mentioned 380 patients were assigned by a data processing supported external random selection of which 198 cases could be evaluated. Tests of significance were executed with a significance level of 5 . Results: Results of the study show a low degree of compliance to the prescribed therapy. However, these therapies were executed in a more structured manner and within a shorter time frame. Measured in terms of the reduction in earning capacity, the outcome analysis shows that only $12.3 \%$ of the treated cases in the intervention group had a reduction in spite of shorter periods of work incapacity. In contrast, a reduction of earning capacity was seen in $27.4 \%$ of the treatment cases in the control group in spite of apparently longer post-treatment periods. Conclusion: Working incapacity time periods and the outcome (reduction of earning capacity) are significantly shorter or, respectively, better within the intervention group. Higher therapy costs do not occur within the intervention group. Studies outlining intensity, duration or value of physiotherapy or occupational therapy have not yet been published. The relevance of structured post-treatment in terms of a combined therapy is therefore underestimated. In particular, prescriptions of occupational therapy are not sufficiently made. Supportive controlling appears to be necessary. Combining both therapies assures a higher treatment success in cooperation with all parties involved.
OP-JOURNAL 2012; 28: 268-271

(c) Georg Thieme Verlag KG Stuttgart · New York DOI http://dx.doi.org/10.1055/s-0032-1327998

\section{Einleitung}

Publikationen zur Behandlung der distalen Radiusfraktur untersuchten im klinischen Bereich in der Regel die Wirksamkeit von chirurgischen Behandlungs- konzepten und deren Outcome; Art, Umfang und Ausmaß des Einsatzes von Übungsbehandlungen standen dabei eher im Hintergrund (z.B. [3-5,7]). Inwieweit therapeutische Lücken zwischen der Akut- und Nachbehandlung 
Auswirkungen auf das Outcome haben können, ist nur unzureichend untersucht worden.

Die aktuelle Übersicht des Cochrane-Reviews zur Rehabilitation bei Erwachsenen mit Frakturen des distalen Radius kommt zu dem Ergebnis, dass die geeignete Form der Nachbehandlung nicht mit ausreichender Evidenz erwiesen ist [1].

Die nachfolgende Arbeit untersuchte u.a. die Frage, welchen Stellenwert die strukturierte postoperative Übungsbehandlung auf das Outcome der distalen Radiusfraktur hat. Das Outcome war dabei als die Dauer der Arbeitsunfähigkeit und die Höhe der Minderung der Erwerbsfähigkeit (MdE) aus der gesetzlichen Unfallversicherung definiert.

\section{Hauptteil}

Die Studie wurde als Zweiarmstudie (Kontroll- und Interventionsgruppe) durchgeführt. Eingeschlossen waren kaufmännische oder gewerbliche Arbeitnehmer, Zeitarbeitnehmer, Umschüler und sonstige Versicherte im Alter zwischen 16 und 65 Lebensjahren mit einer isolierten distalen Radiusfraktur.

In der Kontrollgruppe wurden die Steuerung und Überwachung des Heilverfahrens sowie das Verordnen von Art, Intensität und Ausmaß der Übungsbehandlung ausschließlich dem behandelnden D-Arzt überlassen.

In der Interventionsgruppe wurden nach Eingang des Durchgangsarztberichts und ggf. des OP-Berichts die Unterlagen dem beratenden Handchirurgen übermittelt. Dieser gab innerhalb von 24 Stunden eine dezidierte Behandlungsempfehlung für den weiterbehandelnden Arzt ab, die insbesondere auf die zielgerichtete Verordnung von Physio- und Ergotherapie hinsichtlich Beginn, Art und Dauer abgestellt hat. Voraussichtliche Arbeitsunfähigkeitszeiten werden prognostisch angegeben [2]. Die ausführenden Reha-Manager leiteten diese Empfehlungen an den Behandler weiter, informierten und berieten die Patienten entsprechend und überwachten die Umsetzung.

Die Frakturen wurden entsprechend der Klassifikation der AO klassifiziert sowie die Daten der durchgeführten Übungsbehandlungen erhoben. Diese Werte wurden mit dem Outcome, definiert als Höhe und Häufigkeit einer Minderung

Tab. 1 Verordnungsverhalten von Physiotherapie in IG und KG.

\section{Physiotherapie}

\begin{tabular}{llllll}
\hline & IG & \multicolumn{5}{c}{ KG } & \\
\hline & Mittelwert & Std.-Abw. & Mittelwert & Std.-Abw. & p-Wert \\
\hline $\begin{array}{l}\text { Beginn (Tage } \\
\text { nach Unfall) }\end{array}$ & 34,4 & 16,2 & 32,2 & 18,8 & 0,303 \\
\hline $\begin{array}{l}\text { Ende (Tage } \\
\text { nach Unfall) }\end{array}$ & 78,3 & 48,4 & 124,9 & 169,9 & 0,010 \\
\hline Einheiten & 28,8 & 29,1 & 46,0 & 52,9 & 0,068 \\
\hline Kosten in $€$ & 354,43 & 348,58 & 576,33 & 603,59 & 0,006
\end{tabular}

* U-Test nach Mann-Whitney

der Erwerbsfähigkeit (MdE) sowie der Dauer der Arbeitsunfähigkeit verglichen.

Die Signifikanztests wurden mit einem Signifikanzniveau von 5\% durchgeführt.

\section{Studiengröße und Demografie}

Im Beobachtungszeitraum konnten 380 Patienten randomisiert werden. Aufgrund unterschiedlicher Drop-outs bildeten 198 Patienten, aufgeteilt in beide Gruppen, die empirische Basis der Studie.

Die auf die Studienarme bezogene Verteilung von Alter, Geschlecht und Frakturklassifikation war homogen und zeigte keine signifikanten Unterschiede zwischen den Gruppen.

\section{Übungsbehandlung im Gruppenvergleich}

Die Verordnung von Maßnahmen der Physiotherapie über die Gruppen hinweg stellte sich als bekannte Größe bei der distalen Radiusfraktur dar. Das Verordnungsverhalten von Maßnahmen der Ergotherapie war gruppenspezifisch unterschiedlich. Dabei war zu beobachten, dass auch in der Interventionsgruppe trotz entsprechender Empfehlungen durch den beratenden Arzt Fallgestaltungen vorkamen, in denen weder Physio- noch Ergotherapie verordnet worden war.

Sowohl bei der konservativ als auch bei der operativ behandelten distalen Radiusfraktur sollte immer Physiotherapie, etwa $a b$ der 6.-7. Woche, verordnet werden.

In der Kontrollgruppe wurde in rund 21,3\% der vorliegenden Radiusfrakturen weder Physiotherapie noch Ergotherapie verordnet und durchgeführt. Ein Schwer- punkt hinsichtlich der Frakturklassifikationen war nicht festzustellen. Lediglich bei den C3-Frakturen wurde immer Physiotherapie verordnet.

In der Interventionsgruppe wurde in rund 14,9\% der Fälle keinerlei Übungsbehandlung verordnet. Ein Schwerpunkt der ohne Übungsbehandlung gebliebenen distalen Radiusfrakturen war bei den A2- und B1-Frakturen festzustellen.

Während der Beginn der Physiotherapie, gemessen in Tagen nach dem Unfall, in beiden Gruppen nahezu gleich war, waren beim Ende deutliche Unterschiede festzustellen. Ebenso deutlich unterschied sich die Anzahl der verordneten Einheiten in den Gruppen, auch wenn hier das Signifikanzniveau nicht ganz erreicht wurde. Soweit Übungsbehandlung verordnet wurde, zeigt die Übersicht der Tab. 1 das Verordnungsverhalten in der Kontroll- und Interventionsgruppe. Als Therapieeinheit war definiert, dass an diesen Tagen entsprechende Übungsbehandlung stattgefunden hat.

Während sich die Verordnung von Physiotherapie noch als bekannte Größe darstellte, waren bei dem Verordnungsverhalten von Ergotherapie massive Unterschiede zwischen der Kontroll- und der Interventionsgruppe zu beobachten.

Der Beginn der, soweit überhaupt verordneten, Ergotherapie in der Kontrollgruppe lag im Mittelwert bei 100 Tagen und damit über 50 Tage später als in der Interventionsgruppe. Ähnlich verhielt es sich mit dem Ende der verordneten Ergotherapie. Auch bei der Anzahl der verordneten Einheiten Ergotherapie sowie bei der Kostenstruktur waren signifikante Vorteile zugunsten der Interventionsgruppe zu beobachten. Einzelheiten zeigt Tab. 2. 
Tab. 2 Verordnungsverhalten von Ergotherapie in IG und KG.

\begin{tabular}{|c|c|c|c|c|c|}
\hline & \multicolumn{5}{|c|}{ Ergotherapie } \\
\hline & IG & & KG & & \\
\hline & Mittelwert & Std.-Abw. & Mittelwert & Std.-Abw. & p-Wert* \\
\hline $\begin{array}{l}\text { Beginn (Tage } \\
\text { nach Unfall) }\end{array}$ & 44,2 & 19,3 & 100,1 & 102,7 & 0,016 \\
\hline $\begin{array}{l}\text { Ende (Tage } \\
\text { nach Unfall) }\end{array}$ & 85,1 & 49,5 & 139,0 & 112,4 & 0,028 \\
\hline Einheiten & 27,7 & 63,4 & 32,5 & 32,0 & 0,022 \\
\hline Kosten & 426,73 & 322,21 & 689,86 & 537,44 & 0,031 \\
\hline
\end{tabular}

* U-Test nach Mann-Whitney

Eine kombinierte Verordnung von Physiotherapie und Ergotherapie im Sinne einer multimodalen Nachbehandlungsstrategie war in der Kontrollgruppe die Ausnahme.

Bei der Nachbehandlung der distalen Radiusfraktur sollte neben der Physiotherapie auch Ergotherapie etwa ab der 6.7. Woche regelhaft verordnet werden.

In der Interventionsgruppe war dies wesentlich häufiger der Fall (56\%); festzustellen war, dass die Bereitschaft zur kombinierten Verordnung von Physiound Ergotherapie mit der Dauer der Studie zunahm.

\section{Dauer der Arbeitsunfähigkeit und Minderung der Erwerbsfähigkeit}

Die Dauer der Arbeitsunfähigkeit als Outcomeparameter ergibt sich aus Tab. 3; danach war die mittlere Dauer der Arbeitsunfähigkeit in der Interventionsgruppe bei allen Frakturtypen kürzer und betrug im Mittel 60,2 Tage und war damit um rund 32 Tage kürzer als in der Kontrollgruppe.

Das Outcome, gemessen in der Minderung der Erwerbsfähigkeit (MdE), zeigte trotz kürzerer Arbeitsunfähigkeitszeiten in der Interventionsgruppe in deutlich weniger Fällen eine MdE von mindestens 20 von 100. In der Kontrollgruppe war in $27,4 \%$ eine Rente, wenn z.T. auch nur vorübergehend, festzustellen; in der Interventionsgruppe hingegen nur in $12,3 \%$ der Fälle.

Davon waren in der Kontrollgruppe $19,1 \%$ und in der Interventionsgruppe 9,7\% Gesamtvergütungen (Ges.-Verg.). Berufsgruppenbezogene Schwerpunkte waren dabei nicht festzustellen.
Renten auf unbestimmte Zeit (RuZ) waren in der Kontrollgruppe mit 8,3\% und in der Interventionsgruppe in 2,6\% der Fälle zu gewähren.

Die Verordnung von Physiotherapie bei der Nachbehandlung der distalen Radiusfraktur stellt sich als bekannte Größe dar - die Verordnung von Ergotherapie bei der Nachbehandlung der distalen Radiusfraktur ohne steuernde Eingriffe scheint nicht die Regel zu sein.

In der Interventionsgruppe wurde über die gesamte Behandlungszeit durchschnittlich weniger Übungsbehandlung erbracht als in der Kontrollgruppe. Betrachtet man Intensität als Einheiten von Übungsbehandlung (kombinierte Physio- und Ergotherapie) innerhalb eines bestimmten, möglichst kurzen Zeitraums, bestätigen die gefundenen Ergebnisse eine intensivere Nachbehandlung in der Interventionsgruppe.

Über den gesamten Fallverlauf wurde in der Kontrollgruppe mehr an Übungsbehandlungen verordnet als in der Interventionsgruppe, allerdings über einen längeren Zeitraum. Damit waren die Kosten der Übungsbehandlung in der Interventionsgruppe nicht höher.

Bei der Analyse der Kosten und der Intensität der durchgeführten Physio- und Ergotherapie waren deutliche Einflüsse des Case-Managements zu erkennen. Zwar zeigte sich die Verordnung von Physiotherapie in beiden Gruppen als bekannte Größe, allerdings war in der KG die Verordnung unstrukturierter. Sowohl das durchschnittliche Ende der durchgeführten Therapie in Tagen nach dem Unfall als auch die Anzahl der verordneten Einheiten wichen deutlich von den Daten in der IG ab. Noch deutlicher erschienen die Ergebnisse bei der Ver-
Tab. 3 Dauer der Arbeitsunfähigkeit im Mittelwert.

\begin{tabular}{|c|c|c|}
\hline & \multicolumn{2}{|c|}{ Dauer der Arbeitsunfähigkei } \\
\hline & Mittelwert & Std.-Abw. \\
\hline \multicolumn{3}{|c|}{ AU-Tage Gesamt } \\
\hline KG & 92,6 & 105,8 \\
\hline IG & 60,2 & 38,8 \\
\hline \multicolumn{3}{|c|}{ AU-Tage A-Frakturen } \\
\hline KG & 91,2 & 147,3 \\
\hline IG & 49,5 & 36,3 \\
\hline \multicolumn{3}{|c|}{ AU-Tage B-Frakturen } \\
\hline KG & 64,9 & 39,8 \\
\hline IG & 48,3 & 19,2 \\
\hline \multicolumn{3}{|c|}{ AU-Tage C-Frakturen } \\
\hline KG & 113,5 & 106,6 \\
\hline IG & 81,6 & 45,2 \\
\hline
\end{tabular}

ordnung von Ergotherapie. Der durchschnittliche Beginn von Ergotherapie (in Tagen) in der KG lag nach dem durchschnittlichen Ende der Ergotherapie in der IG. Sowohl der Beginn von Physioals auch Ergotherapie erschienen spät im Verlauf, spiegelten aber die Realität des Behandlungsbeginns wider. Nur mit diesen unterstützenden Maßnahmen konnten die funktionellen Folgen im Sinne der Bewegungseinschränkung im Handgelenk im Vergleich zur unverletzten Seite so gering wie möglich gehalten werden. Allein die Rentenquote in den jeweiligen Gruppen dürfte die Wirksamkeit der Nachbehandlung in der Behandlungskette untermauern. Die Ergebnisse dieser Untersuchung konnten den Stellenwert konsequent durchgeführter Nachbehandlung untermauern.

Betrachtet man die Dauer der Arbeitsunfähigkeit, sind die Einflüsse aus der Interventionsgruppe, das Case-Management als auch die Nachbehandlungsintensität deutlich zu erkennen. Allerdings wird die Schlussfolgerung, die kürzere Dauer der Arbeitsunfähigkeit allein dem Ausmaß der intensiveren Nachbehandlung zuzurechnen, nicht zulässig sein. Hier sind die steuernden Einflüsse ebenso zu berücksichtigen wie die durch die intensivere Übungsbehandlung erreichte bessere Funktion der Hand.

Durch die kombinierte Verordnung von Physio- und Ergotherapie kann ein besseres Outcome erreicht werden. 
Bei der Minderung der Erwerbsfähigkeit (MdE) konnte nachgewiesen werden, dass das Outcome in der Interventionsgruppe signifikant besser ist als in der Kontrollgruppe. Das Outcome in der Kontrollgruppe entspricht weitestgehend auch den bisher publizierten Feststellungen anderer Unfallversicherungsträger [6]. Die strukturierte Nachbehandlung im Sinne einer Kombination aus Physio- und Ergotherapie sowie abschwellender Maßnahmen scheint in ihrer Wirkung noch unterschätzt zu werden. Die Ergebnisse in der Interventionsgruppe belegen allerdings eindrucksvoll, welches Kosten-Nutzen-Verhältnis mit dieser Art der kombinierten Therapie zu erreichen ist.

Die hypothetisch angenommenen niedrigeren Gesamtfallkosten in der Interventionsgruppe ließen sich bereits ohne ökonomische Erhebungen durch die kürzeren Arbeitsunfähigkeitszeiten und niedrigen Rentenquoten verifizieren.

\section{Schlussfolgerung}

Die strukturierte Nachbehandlung im Sinne einer kombinierten Physio- und Ergotherapie bei der distalen Radiusfrak- tur wird in ihrer Bedeutung noch unterschätzt.

Insbesondere bei der Verordnung von Ergotherapie fallen deutliche Defizite auf. Eine unterstützende Steuerung scheint daher geboten. Als Therapieempfehlungen hinsichtlich Beginn, Art und Dauer der Nachbehandlung bieten sich dazu z. B. die Standards für Heilverfahren und Rehabilitation [8] der VBG an. Die Kombination beider Therapieverfahren sichert im Zusammenwirken aller Beteiligten einen größeren Behandlungserfolg. Die interdisziplinäre Zusammenarbeit zwischen UV-Trägern, Ärzten und Therapeuten ist dabei in einem höheren Maße gefordert als bisher.

Das Outcome, gemessen als Dauer der Arbeitsunfähigkeit als auch als Minderung der Erwebsfähigkeit (MdE), ist in der Interventionsgruppe signifikant besser. Höhere Therapiekosten sind, auch ohne Budgetschranken, in der Interventionsgruppe nicht zu beobachten. Untersuchungen, die Intensität, Dauer und Wert von Physio- und Ergotherapie beleuchten, sind bisher nicht publiziert.

\section{Literatur}

${ }^{1}$ Handholl HHG, Madhok R, Howe TE. Rehabilitation for distal radial fractures in adults. Cochrane Review 2006; 3

2 Lohsträter A, Schmidt J. Rehabilitationsmanagement bei Hand- und Handgelenksverletzungen aus Sicht des UV-Trägers. Trauma Berufskrankh 2002; 4: 163-167

3 Oestern H. Aktueller Stand der Behandlung von Radiusfrakturen. Trauma Berufskrankh 2003; 5 (Suppl. 1): S22-S25

4 Pabst T, Uzdil T, Winker KH. Die palmare Plattenosteosynthese bei der distalen Radiusfraktur. OP-Journal 2003; 19: 42-50

${ }^{5}$ Pilz F, Lindemann-Sperfeld $L$, Winter $S$ et al. Distale Radiusfrakturen - Behandlungskonzepte und Erfahrungen. Trauma Berufskrankh 2000; 2: 313-319

${ }^{6}$ Radek E, Köhler T, Münch $K$ et al. Verletztenrenten auf unbestimmte Zeit nach distalen Radiusfrakturen. Trauma Berufskrankh 2003; 5: 253-259

7 Siebert H, Klonz A. Distale Radiusfraktur. Unfallchirurg 2005; 108: 135-154

8 VBG. Standards für Heilverfahren und Rehabilitation. Hamburg: VBG; 2007: 59-64

Dr. rer. med. Axel Lohsträter Leitung Bezirksverwaltung

\section{Silvia Germann}

Rehakoordinator

VBG (Verwaltungsberufsgenossenschaft) Koenbergkstraße 1 99084 Erfurt

axel.lohstraeter@vbg.de 